\title{
Particle Filter for Estimating Freeway Traffic State in Beijing
}

\author{
Jun Bi, ${ }^{1}$ Can Chang, ${ }^{1}$ and Yang Fan ${ }^{2}$ \\ ${ }^{1}$ School of Traffic and Transportation, Beijing Jiaotong University, Beijing 100044, China \\ ${ }^{2}$ Department of Civil and Environmental Engineering, University of Wisconsin-Madison, Madison, WI 53706, USA
}

Correspondence should be addressed to Jun Bi; bilinghc@163.com

Received 15 July 2013; Revised 10 October 2013; Accepted 24 October 2013

Academic Editor: Wuhong Wang

Copyright (C) 2013 Jun Bi et al. This is an open access article distributed under the Creative Commons Attribution License, which permits unrestricted use, distribution, and reproduction in any medium, provided the original work is properly cited.

\begin{abstract}
Freeway traffic state estimation is useful for intelligent traffic guidance, control, and management. The freeway traffic state is featured with rapid and dramatic fluctuations, which presents a strong nonlinear feature. In theory, a particle filter has good performance in solving nonlinear problems. This paper proposes a particle filter based approach to estimate freeway traffic state. The freeway link between the west of Peace Bridge and the west of San Yuan Bridge of the third ring in Beijing is used as the experimental object. According to the traffic characteristics and measurement mode of the link, the second-order validated macroscopic traffic flow model is adopted to set up the link model. The implementation steps of the particle filter for freeway traffic state estimation are described in detail. The estimation error analysis for the experiments proves that the proposed approach has an encouraging estimation performance.
\end{abstract}

\section{Introduction}

The increasing traffic flow is resulting in serious congestion of urban road network, which can decrease flow rate, delay travel time, increase fuel consumption and travel cost, and make negative environmental effect. Measures should be taken to alleviate traffic congestion. Traffic state estimation of freeway network is useful for traffic management, which involves estimating the traffic variables of the network based on available real-time traffic measurements [1]. A real-time Lagrangian traffic state estimator for freeway state has been proposed, which is considered to be more accurate and more computationally efficient than the Eulerian approach $[2,3]$. However, the Lagrangian method is only appropriate for the sensing data obtained via GPS technology or other tracking devices providing position and velocity of individual vehicles. Chao Deng et al. present an approach using cluster analysis and multiclass support vector machine to estimate freeway traffic state. Historical traffic flow data are divided into clusters with different traffic states and the multiclass support vector machine is applied to identify the real-time traffic states [4]. Many previous researches in estimating aggregated traffic variables are based on applications of Kalman filter or extended Kalman filter [5-7]. Furthermore, most of them assume that noises obey Gaussian distribution and use linear models for state functions and observation functions. However, traffic state can fluctuate rapidly and dramatically in a short time, which indicates the strong nonlinear features of the freeway traffic state. Therefore, using Kalman filter may cause inaccurate estimation results and even divergent results. Particle filter is a powerful approximate solution to a general nonlinear problem or a non-Gaussian filtering problem $[8,9]$. The basic idea of particle filter is that a posterior probability density function (PDF) of state can be represented by a set of particles with associated weights, and the estimation can be computed as the expected value of the discrete PDF $[10,11]$. Currently only limited number of papers have discussed freeway traffic state estimation using particle filter algorithm. Mihaylova and Boel [12] proposed a particle filter (PF) to estimate freeway traffic based on the model of aggregated states and observations, and the investigations are conducted using the real traffic data from a Belgian freeway. Nicolae Marinica developed a particle filter (PF) state estimator using a platoon based model for urban traffic networks [13]. This paper uses the second-order validated macroscopic traffic flow model to evaluate a freeway link in Beijing according to its characteristics and proposes a particle filter method for estimating the freeway speed and density.

The rest of the paper is organized as follows. Section 2 describes a freeway traffic flow model in Beijing. Section 3 
presents the design of the traffic state estimator based on the particle filter algorithm. In Section 4, several experiments are conducted to evaluate the particle filter estimation performance. Finally, the conclusions are summarized in Section 5.

\section{Traffic Flow Modeling of a Freeway}

2.1. Description of a Freeway. A freeway network can be represented as a directed graph. Bifurcations, junctions, onramps, and off-ramps of a freeway are represented as nodes, and a freeway stretch between two nodes is represented as a link in the graph [12]. A bidirectional freeway stretch is modeled as two opposite directional links. As shown in Figure 1, the freeway is divided into several links and each link is composed of several sections. In the paper, each link is assumed to have homogeneous geometric characteristics such as the number of lanes, slopes, and curvatures. Detectors are available only at some boundaries between sections. In Figure $1, q_{i}$ is the average number of vehicles at the boundary between sections $i$ and $i+1, d_{i}$ is the average density in section $i, v_{i}$ is the average speed in section $i, s_{i}$ is the traffic flow into ramp $i$, and $r_{i}$ is the traffic flow off ramp $i$. The whole state of the link $m$ at time $k$ is described by the vector $x_{k}=\left(x_{1, k}^{T}, x_{2, k}^{T}, \ldots, x_{i, k}^{T}\right)^{T}$, and $x_{i, k}=\left(v_{i, k}, d_{i, k}\right)^{T}$ is a local state vector of the $i$ th section.

The progression from one sampling time to the next sampling time is described by the update equation

$$
x_{k+1}=f\left(x_{k}, p_{k}, q_{k}^{\text {In }}, v_{k}^{\text {in }}, q_{k}^{\text {out }}, v_{k}^{\text {out }}, \xi_{k}\right) \text {, }
$$

where $p_{k}$ denotes the vector of all time-varying parameters such as road conditions and the number of available lanes, $q_{k}^{\text {in }}$ is the number of vehicles entering Section 1 during the interval $\left[t_{k}, t_{k+1}\right), v_{k}^{\text {in }}$ is the average speed of these vehicles, $q_{k}^{\text {out }}$ specifies the outflow at the speed $v_{k}^{\text {out }}$ from section $N$, and $\xi_{k}$ is a disturbance vector reflecting random fluctuation in the traffic states and the model error.

Noisy measurements of the average speed of vehicles crossing the boundary between section $i$ and section $i+1$ during the time interval $\left[t_{k}, t_{k+1}\right)$ together with noisy measurements of the mean density of the vehicles are collected as the measurement data. The observation equation is given as

$$
y_{k+1}=h\left[x_{s}, \eta_{k}, s \in\left[t_{k}, t_{k+1}\right)\right],
$$

where $x_{s}$ is the state at time $s$ and $\eta_{k}$ is the measurement noise.

2.2. Macroscopic Traffic Flow Model of a Freeway. The secondorder validated macroscopic traffic flow model is employed to describe the dynamic behavior of traffic flow along a freeway stretch in terms of appropriate aggregated traffic variables such as traffic density, space average speed, and traffic flow [14]. Generally, a freeway stretch is divided into a number of $N$ sections. Assume that the length of each section is $L_{i}$, $i=1,2, \ldots, N$, the time discretization is based on a time step $T$, and $k(k=0,1,2, \ldots)$ is a discrete time index. For the section $i$, the stochastic nonlinear difference equations

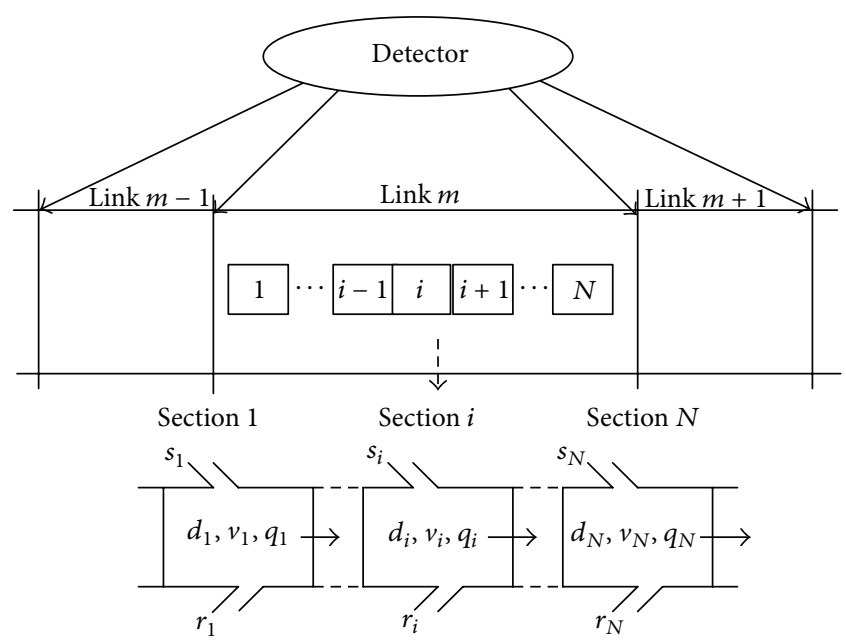

FIGURE 1: Freeway links and measurement points.

based on the second-order macroscopic traffic flow model are described as follows [14]:

$$
\begin{aligned}
d_{i}(k+1)=d_{i}(k) & +\frac{T}{\lambda_{i} L_{i}} \\
\times & {\left[q_{i-1}(k)-q_{i}(k)+r_{i}(k)-s_{i}(k)\right], } \\
s_{i}(k)=\beta_{i}(k) q_{i-1}(k), & \\
v_{i}(k+1)= & v_{i}(k)+\frac{T}{\tau}\left[v\left(d_{i}(k)\right)-v_{i}(k)\right] \\
& +\frac{T}{L_{i}} v_{i}(k)\left[v_{i-1}(k)-v_{i}(k)\right] \\
& -\frac{n T}{\tau L_{i}} \frac{\left[d_{i+1}(k)-d_{i}(k)\right]}{d_{i}(k)+\kappa} \\
& -\frac{\delta T}{\lambda_{i} L_{i}} \frac{r_{i}(k) v_{i}(k)}{d_{i}(k)+\kappa}+\xi_{i}^{v}(k), \\
v(d)= & v_{f} \exp \left[-\frac{1}{a}\left(\frac{d}{d_{\mathrm{cr}}}\right)^{a}\right], \\
q_{i}(k)= & d_{i}(k) v_{i}(k) \lambda_{i}+\xi_{i}^{q}(k) .
\end{aligned}
$$

In (3), the variables and parameters are denoted as follows:

(1) traffic density $d_{i}(k)$ (in veh/ $\mathrm{km} /$ lane) is the number of vehicles in section $i$ at time $k T$ divided by the section length $L_{i}$ and the number of lanes $\lambda_{i}$,

(2) space average speed $v_{i}(k)$ (in $\mathrm{km} / \mathrm{h}$ ) is the average speed of the vehicles in section $i$ at time $k T$,

(3) traffic flow $q_{i}(k)$ (in veh/h) is the number of vehicles leaving section $i$ during the interval $[k T,(k+1) T)$, divided by $T$,

(4) $r_{i}(k)$ is the on-ramp inflow and $s_{i}(k)$ is the off-ramp outflow (both in veh/h) in section $i$. 
(5) $\tau, n, \kappa, a$, and $\lambda$ are the model parameters which are the same given values for all sections,

(6) $v_{f}$ denotes the free flow speed, $d_{c r}$ is the critical density, and $a$ is the exponent of the stationary speed equation,

(7) $\xi_{i}^{v}(k)$ and $\xi_{i}^{q}(k)$ reflect the model error, $\xi_{i}^{v}(k)$ denotes the zero-mean Gaussian white noise acting on the empirical speed equation, and $\xi_{i}^{q}(k)$ denotes the zeromean Gaussian white noise acting on the approximate flow equation.

The detectors in the link entrance or exit can collect the speed data and density data. The speed measurement function is

$$
Z_{\text {in or out }}^{v}(k)=v_{\text {in or out }}(k)+\xi_{\text {in or out }}^{v}(k),
$$

where $Z_{\text {in or out }}^{v}(k)$ is the measurement value of the average speed through entrance or exit during the interval $[(k-$ $1) T, k T)$ and $\xi_{\text {in or out }}^{v}(k)$ is the measurement noise of the speed.

The measurement function of the density for the link entrance or exit is

$$
Z_{\text {in or out }}^{d}(k)=d_{\text {in or out }}(k)+\xi_{\text {in or out }}^{d}(k),
$$

where $Z_{\text {in or out }}^{d}(k)$ is the measurement value of the mean density through entrance or exit during the interval $[(k-1) T, k T)$ and $\xi_{\text {in or out }}^{d}(k)$ is the measurement noise of the density.

\section{Particle Filter for Freeway Traffic State Estimation}

Nonlinear characteristics exist in the freeway traffic state, which makes it difficult to estimate the traffic state. Since particle filter is able to solve a general nonlinear problem in theory, particle filter is well studied to estimate the traffic state based on the second-order validated macroscopic traffic flow model.

3.1. Particle Filter Theory. The discrete-time stochastic model of a dynamic system is described as follows:

$$
\begin{gathered}
x_{k}=f_{k}\left(x_{k-1}, w_{k-1}\right), \\
y_{k}=h_{k}\left(x_{k}, v_{k}\right),
\end{gathered}
$$

where $y_{k}$ is the observation vector at time $k, x_{k}$ represents the state vector at time $k, f_{k}$ is the nonlinear state transition function, and $h_{k}$ is the nonlinear observation function. The stochastic processes $w_{k}$ and $v_{k}$ represent the state noise process and the measurement noise process, respectively. The available information at time $k$ is the set of measurements $Z_{k}=\left\{y_{i}: i=1,2, \ldots, k\right\}$. According to the Bayesian theory, all state information can be obtained from the posterior state distribution. Within the Bayesian framework, particle filter is used to sequentially update a priori knowledge about predetermined state vector $x_{k}$ by using the measurements data $Z_{k}$. Suppose that the initial probability density function of the state vector is $p\left(x_{0} \mid Z_{0}\right)=p\left(x_{0}\right)$; then we have $[9,10]$

$$
\begin{gathered}
p\left(x_{k} \mid Z_{k-1}\right)=\int p\left(x_{k} \mid x_{k-1}\right) p\left(x_{k-1} \mid Z_{k-1}\right) d x_{k-1}, \\
p\left(x_{k} \mid Z_{k}\right)=\frac{p\left(y_{k} \mid x_{k}\right) p\left(x_{k} \mid Z_{k-1}\right)}{p\left(y_{k} \mid Z_{k-1}\right)} \\
p\left(y_{k} \mid Z_{k-1}\right)=\int p\left(y_{k} \mid x_{k}\right) p\left(x_{k} \mid Z_{k-1}\right) d x_{k} .
\end{gathered}
$$

Particle filter uses Monte Carlo techniques and sequence importance sampling (SIS) methods to solve (7). The posterior PDF is computed based on $N$ samples from an important distribution function, as follows,

$$
\begin{gathered}
p\left(x_{k} \mid Z_{k}\right)=\sum_{i=1}^{N} w_{k}^{i} \delta\left(x_{k}-x_{k}^{i}\right), \\
w_{k}^{i}=\frac{\bar{w}_{k}^{i}}{\sum_{i=1}^{N} \bar{w}_{k}^{i}}, \\
\bar{w}_{k}^{i}=\bar{w}_{k-1}^{i} \frac{p\left(y_{k} \mid x_{k}^{i}\right) p\left(x_{k}^{i} \mid x_{k-1}^{i}\right)}{q\left(x_{k}^{i} \mid x_{k-1}^{i}, y_{k}\right)},
\end{gathered}
$$

where $w_{k}^{i}$ is the weight of each particle and satisfies $0 \leq w_{k}^{i} \leq$ 1 and $q$ is an important distribution function.

Particle filter has good effects in solving both nonlinear and non-Gaussian applications, which is suitable for estimating the nonlinear freeway state.

3.2. Application to Freeway Traffic State Estimation. The application steps of freeway traffic state estimation based on particle filter are as follows.

(1) Initialization. Set the iteration variable $k=0$. The particle set $\left\{x_{0}^{i}, w_{0}^{i}\right\}(i=0,1,2, \ldots, N, N$ is the number of particles $)$ is obtained based on the initial distribution $p\left(x_{0}\right)$.

(2) Particle Generation. For $i=1,2, \ldots, N, x_{k}^{i}$ is sampled from $p\left(x_{k} \mid x_{k-1}^{i}, Z_{k}\right)$ according to (3).

(3) Weight Computation. Equation (12) is complex to compute the weight, and usually the weight is updated by the following equation when the new measurement data are obtained by the detector in the section:

$$
\bar{w}_{k}^{i}=\bar{w}_{k-1}^{i} \times p\left(y_{k} \mid x_{k}^{i}\right) .
$$

The normalized weight $w_{k}^{i}$ is computed using (11).

(4) Resampling. Drawing a random sample $u_{i}$ from the uniform distribution over $(0,1]$. If $u_{i}$ satisfies $\sum_{j=1}^{m-1} w_{k}^{j}<u_{i} \leq$ $\sum_{j=1}^{m} w_{k}^{j}$, the $m$ th particle is resampled as follows:

$$
\begin{aligned}
\tilde{x}_{k}^{i} & =x_{k}^{m}, \\
\widetilde{w}_{k}^{i} & =w_{k}^{m} .
\end{aligned}
$$


(5) State Estimation. The approximate posterior PDF is computed using (10); then the state is estimated by

$$
\widehat{x}_{k}=\sum_{i=1}^{N} \widetilde{w}_{k}^{i} \widetilde{x}_{k}^{i} .
$$

(6) $k$ Is increased by Itself. If the end condition of algorithm is not satisfied, the algorithm goes to step (2) again; otherwise, it ends.

\section{Freeway State Estimation Experiments}

4.1. Experimental Object. A freeway between the west of Peace Bridge and the west of San Yuan Bridge of third ring in Beijing is used as an experimental link. The link has no ramps. The length of the link is $1476 \mathrm{~m}$. The link is composed of 3 sections with 3 lanes and the length of each section is $556 \mathrm{~m}$, $475 \mathrm{~m}$, and $445 \mathrm{~m}$, respectively. The measurement detectors are located in the boundaries between the first section and the third section. The detectors can collect the traffic flow, speed, and density of the link every 2 min.

One example of the entrance traffic state during 3 hours between 8:00 and 11:00 AM on July 23, 2010, is shown in Figure 2. We know that the traffic flow varied between $40 \mathrm{veh} / 2 \mathrm{~min}$ and $60 \mathrm{veh} / 2 \mathrm{~min}$, the speed varied between $32 \mathrm{~km} / \mathrm{h}$ and $50 \mathrm{~km} / \mathrm{h}$, and the density varied between $23 \mathrm{veh} / \mathrm{km}$ and $58 \mathrm{veh} / \mathrm{km}$. In most of the time, the traffic state varied smoothly except for a rapid change at 8:20 AM.

4.2. Experiments and Analysis. The state vector $x_{k}$ and measurement vector $Z_{k}$ are computed, respectively, as follows:

$$
\begin{gathered}
x_{k}=\left[v_{1}(k), d_{1}(k), v_{2}(k), d_{2}(k), v_{3}(k), d_{3}(k)\right]^{T}, \\
Z_{k}=\left[Z_{\text {in }}^{v}(k), Z_{\text {in }}^{d}(k), Z_{\text {out }}^{v}(k), Z_{\text {out }}^{d}(k)\right] .
\end{gathered}
$$

The parameters of traffic model in Section 2 are set as follows.

According to the experimental object in Section 4.1, each section of the freeway has 3 lanes, and the length of each section is about $500 \mathrm{~m}$. The data detection period is $2 \mathrm{~min}$. In daytime, the free flow speed of the freeway is about $80 \mathrm{~km} / \mathrm{h}$ and the critical density is about $50 \mathrm{veh} / \mathrm{km}$. Therefore, some of the parameters can be set as $\lambda_{i}=3, L_{i}=500 \mathrm{~m}, T=$ $120 \mathrm{~s}, v_{f}=80 \mathrm{~km} / \mathrm{h}$, and $d_{\mathrm{cr}}=50 \mathrm{veh} / \mathrm{km}$. Based on the prior knowledge, other parameters can be set as $\tau=20 \mathrm{~s}$, $n=45 \mathrm{~km}^{2} / \mathrm{s}$, and $\kappa=15 \mathrm{veh} / \mathrm{km} /$ lane.

According to the data analysis in Figure 2, the initial state is set to be

$$
x_{0}=[42,40,50,35,48,35]^{T} .
$$

The number of particles $N$ is set to be 500 .

The freeway speed and density are estimated using the particle filter algorithm in the Section 3. The estimation results for the speed and the density of each section are, respectively, shown in Figures 3 and 4 . In the horizontal axis of each figure, the step time interval is $2 \mathrm{~min}$ which is equal to the data collecting interval of the detectors. According to Figures 3 and 4 , the estimation trend is similar to the true trend.
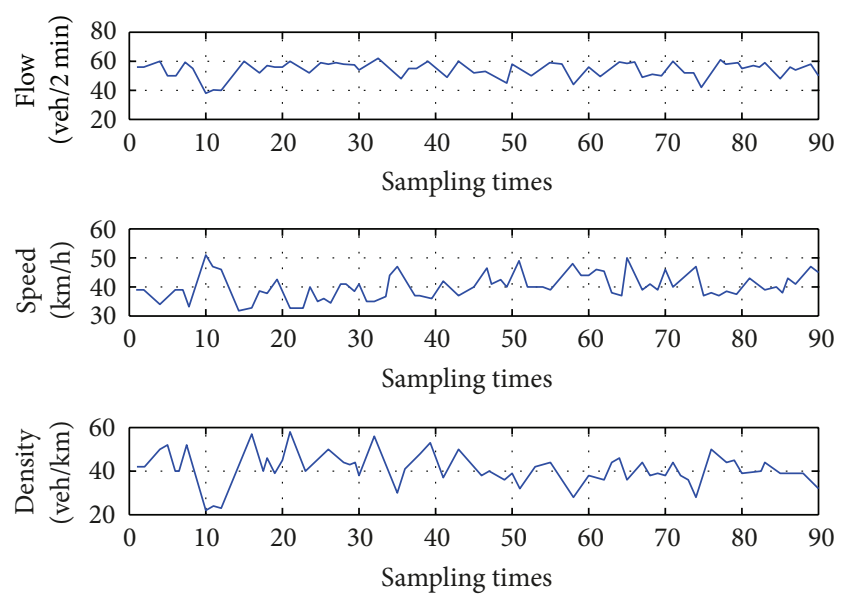

Figure 2: Traffic state example of exit in the link.
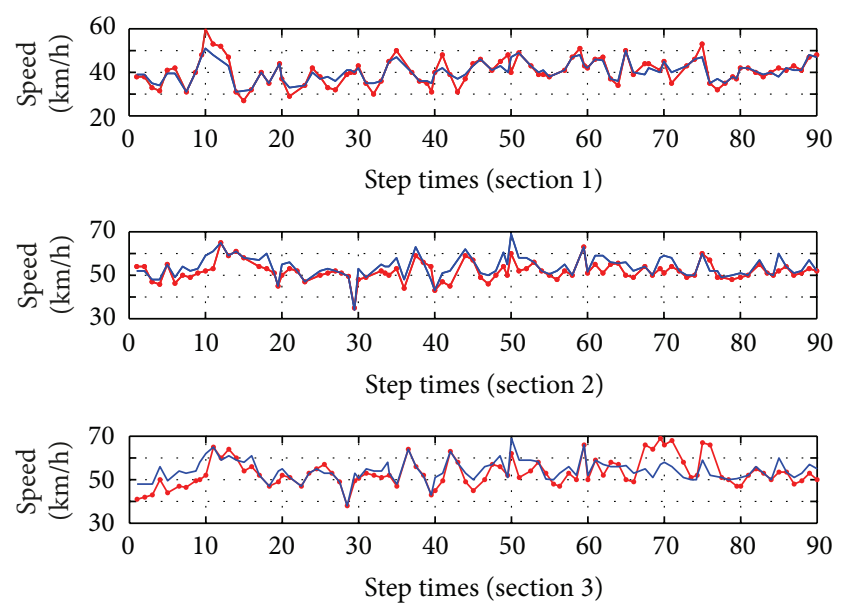

$\begin{array}{ll}\longrightarrow & \text { Estimation } \\ - & \text { True }\end{array}$

FIGURE 3: Speed estimation for three sections.

The indices of relative error (RE), mean square error (MSE), and root mean square relative error (RMSRE) are utilized to evaluate estimation performance.

The indices of RE, MSE, and RMSE are, respectively, defined as follows:

$$
\begin{aligned}
\mathrm{RE} & =\frac{x_{i}-\widehat{x}_{i}}{x_{i}} \times 100 \%, \\
\mathrm{MSE} & =\sqrt{\frac{1}{n} \sum_{i=1}^{n}\left(x_{i}-\widehat{x}_{i}\right)^{2},} \\
\mathrm{RMSRE} & =\sqrt{\frac{1}{n} \sum_{i=1}^{n}\left(\frac{x_{i}-\widehat{x}_{i}}{x_{i}}\right)^{2}},
\end{aligned}
$$

where $x_{i}$ represents the true state and $\widehat{x}_{i}$ represents the estimation state.

The REs of speed estimation and density estimation for each section are, respectively, shown in Figures 5 and 6 . In the horizontal axis of each figure, each step time interval is $2 \mathrm{~min}$. 

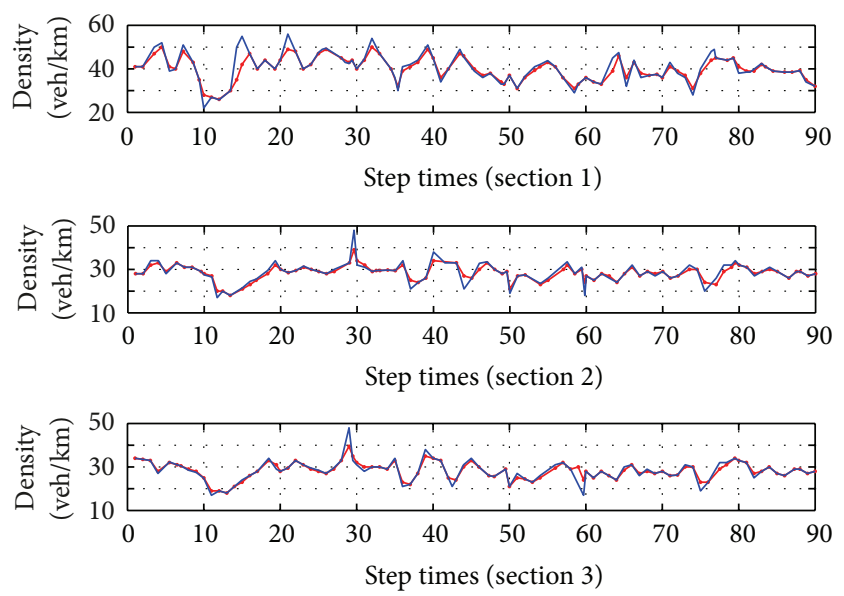

- Estimation

- True

Figure 4: Density estimation for three sections.
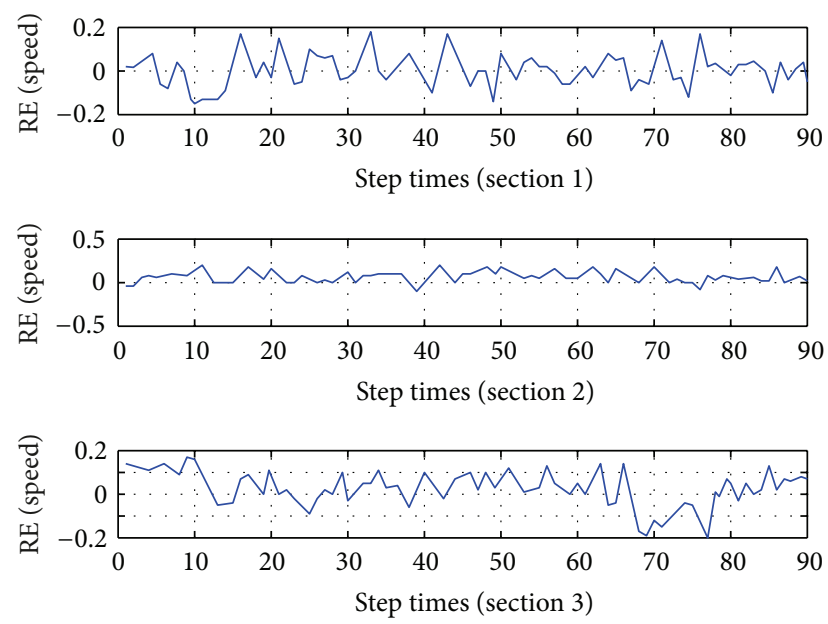

FIgURE 5: REs of speed estimation for three sections.

According to Figures 5 and 6 , the RE is in $[-10 \%,+10 \%]$ and varies smoothly in most of the time. Only at several time intervals, the absolute RE is more than $15 \%$.

The MSE and RMSRE of speed estimation and the MSE and RMSRE of density estimation for each section are shown in Table 1. The maximum MSE difference of speed estimation among the sections is 1.8675 and the maximum RMSRE difference of speed estimation among the sections is 0.0156 . The maximum MSE difference of density estimation among the sections is 1.0273 and the maximum RMSRE difference of density estimation among the sections is 0.0062 . The mean MSE of the speed estimation is 3.7136, the mean RMSRE of the speed estimation is 0.0750 , the mean MSE of the density estimation is 2.6859 , and the mean RMSRE of the density estimation is 0.0751 . Therefore, the estimation effect is desirable.

In theory, the resampling procedure in particle filter has a complexity of $O(N) ; N$ is the number of sample variables generated from the uniform distribution [11]. In fact, the computation time can be reduced in the particle filter implementation by using the multithread programming technology.
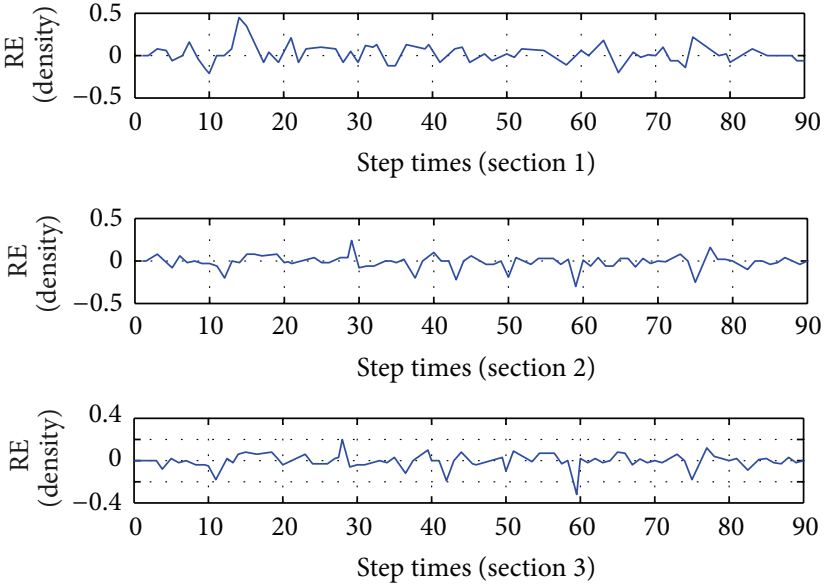

FIGURE 6: REs of density estimation for three sections.

TABLE 1: MSE and RMSRE of estimation of density and speed.

\begin{tabular}{lcccc}
\hline \multirow{2}{*}{ Error index } & \multicolumn{2}{c}{ Speed } & \multicolumn{2}{c}{ Density } \\
& MSE & RMSRE & MSE & RMSRE \\
\hline Section 1 & 2.8269 & 0.0697 & 3.0131 & 0.0786 \\
Section 2 & 3.6196 & 0.0700 & 3.0360 & 0.0742 \\
Section 3 & 4.6944 & 0.0853 & 2.0087 & 0.0724 \\
\hline Mean & 3.7136 & 0.0750 & 2.6859 & 0.0751 \\
\hline
\end{tabular}

\section{Conclusion}

This paper proposes an approach to estimate the freeway traffic state based on the particle filter algorithm. The freeway traffic is modeled by the second-order validated macroscopic traffic flow model. A freeway link between the west of Peace Bridge and the west of San Yuan Bridge of third ring in Beijing is used as an experimental object and real traffic data are used in the experiments. The mean square error of the speed estimation is 3.7136 and the mean square error of the density estimation is 2.6859. The results suggest that particle filter is valid and effective in freeway traffic state estimation. In addition, the proposed approach for traffic state estimation is modular, and therefore different traffic models can be used in different sections on a freeway link. In the future research, we will use parallel computation to improve the performance of the particle filter for estimating freeway state.

\section{Conflict of Interests}

The authors declare that there is no conflict of interests regarding the publication of this paper.

\section{Acknowledgments}

This paper is sponsored by the National 863 Program (2011AA110303) and the Fundamental Research Funds for the Central Universities (2013JBM052). 


\section{References}

[1] Y. B. Wang and M. Papageorgiou, "Real-time freeway traffic state estimation based on extended Kalman filter: a case study," Transportation Science, vol. 41, no. 2, pp. 167-181, 2007.

[2] C. Herrera and A. M. Bayen, "Incorporation of Lagrangian measurements in freeway traffic state estimation," Transportation Research B, vol. 44, no. 4, pp. 460-481, 2010.

[3] Y. F. Yuan, J. W. C. van Lint, R. E. Wilson, and F. van Wageningen-Kessels, "Real-time lagrangian traffic state estimator for freeways," IEEE Transactions on Intelligent Transportation Systems, vol. 13, no. 1, pp. 59-70, 2012.

[4] C. Deng, F. Wang, and H. M. Shi, "Real-time freeway traffic state estimation based on cluster analysis and multiclass support vector machine," in Proceedings of the 1st International Workshop Intelligent Systems and Applications, pp. 1-4, May 2009.

[5] Y. C. Zhao, Y. C. Liu, L. Q. Shan, and B. Zhou, "Dynamic analysis of Kalman filter for traffic flow forecasting in sensornets," Information Technology Journal, vol. 11, no. 10, pp. 1518-1522, 2012.

[6] Y. B. Wang and M. Papageorgiou, "Real-time freeway traffic state estimation based on extended Kalman filter: adaptive capabilities and real data testing," Transportation Research A, vol. 42, no. 10, pp. 1340-1358, 2008.

[7] C. P. I. J. van Hinsbergen, T. Schreiter, and F. S. Zuurbier, "Localized extended kalman filter for scalable real-time traffic state estimation," IEEE Transactions on Intelligent Transportation Systems, vol. 13, no. 1, pp. 385-394, 2012.

[8] K. Nummiaro, E. Koller-Meier, and L. van Gool, "An adaptive color-based particle filter," Image and Vision Computing, vol. 21, no. 1, pp. 99-110, 2003.

[9] A. Doucet, N. de Freitas, and N. Gordon, Eds., Sequential Monte Carlo in Practice, Springer, New York, NY, USA, 2001.

[10] J. Bi, Y. F. Fung, T. K. Ho, and B.H. Mao, "Parallel resampling particle filter algorithm," Journal of Computational Information Systems, vol. 7, no. 6, pp. 1838-1845, 2011.

[11] W. Yi, M. R. Morelande, L. J. Kong, and J. Y. Yang, "A computationally efficient particle filter for multitarget tracking using an independence approximation," IEEE Transactions on Signal Processing, vol. 61, no. 4, pp. 843-856, 2013.

[12] L. Mihaylova and R. Boel, "A particle filter for freeway traffic estimation," in Proceedings of the 43rd IEEE Conference on Decision and Control (CDC '14), pp. 2106-2111, December 2004.

[13] N. Marinica and R. Boel, "Particle filter for platoon based models of urban traffic," in Proceedings of the 15th WSEAS International Conference on Systems, pp. 42-47, July 2011.

[14] M. Papageorgiou, "Some remarks on macroscopic traffic flow modelling," Transportation Research A, vol. 32, no. 5, pp. 323$329,1998$. 


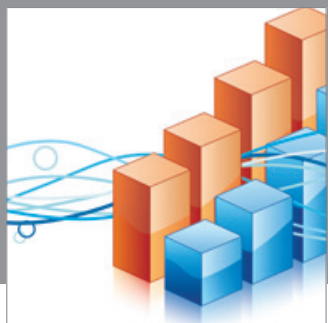

Advances in

Operations Research

mansans

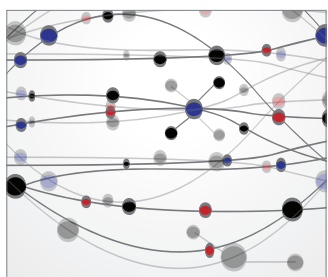

The Scientific World Journal
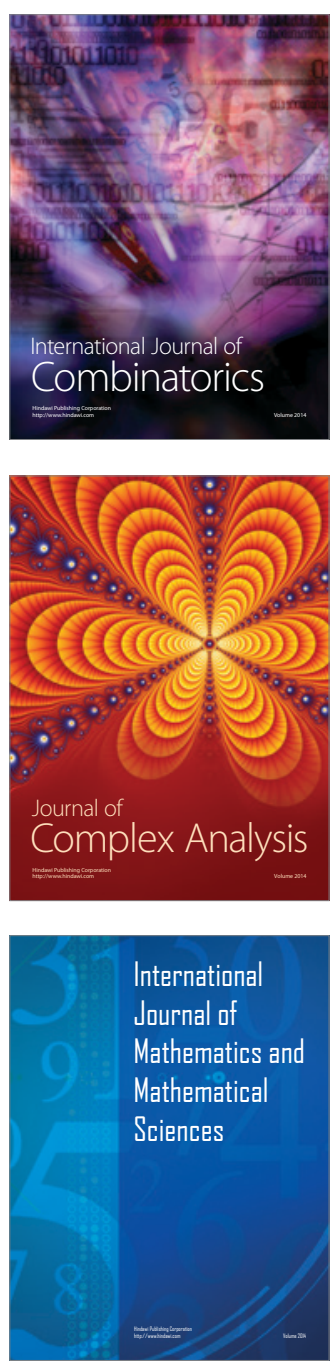
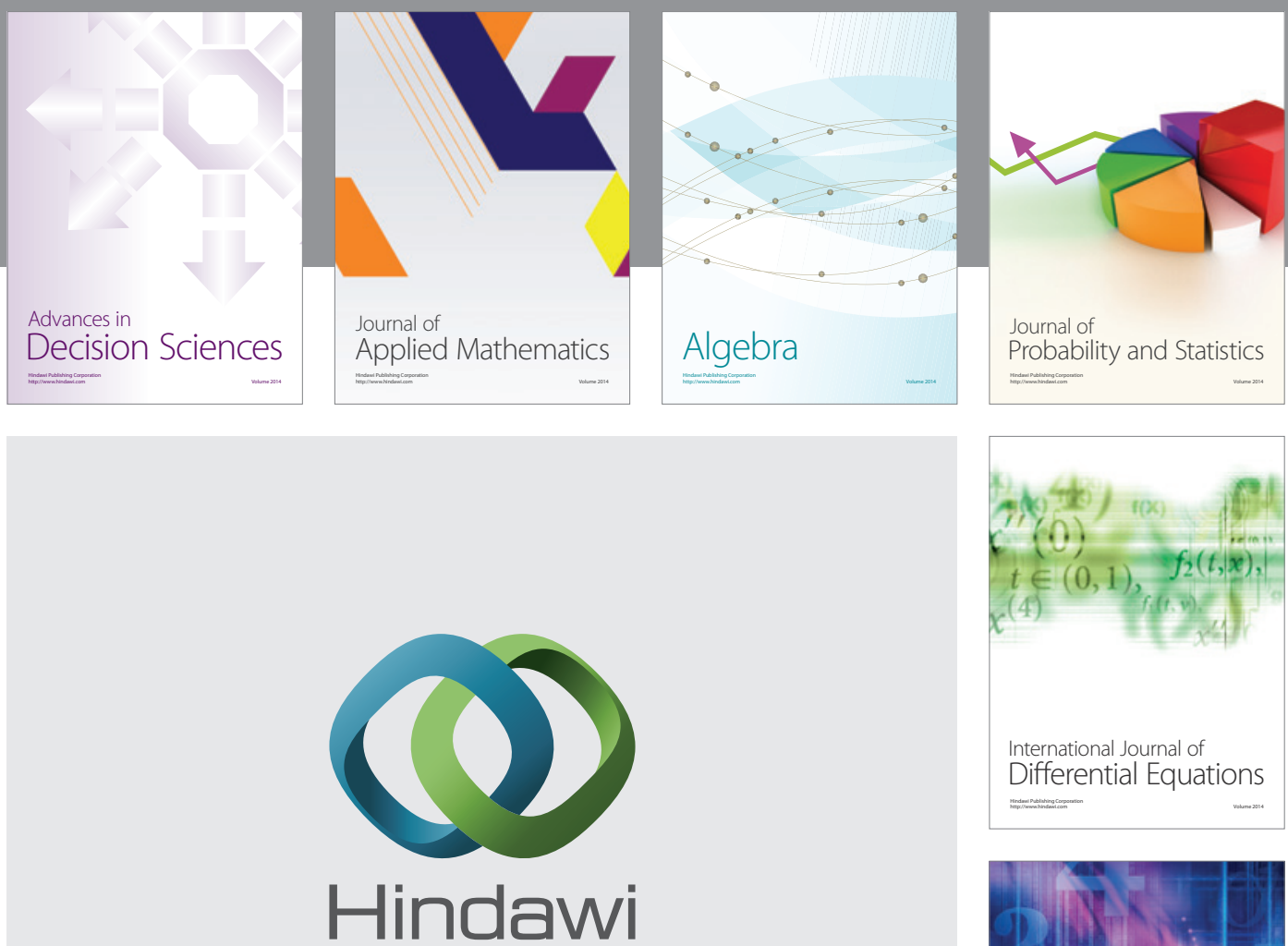

Submit your manuscripts at http://www.hindawi.com
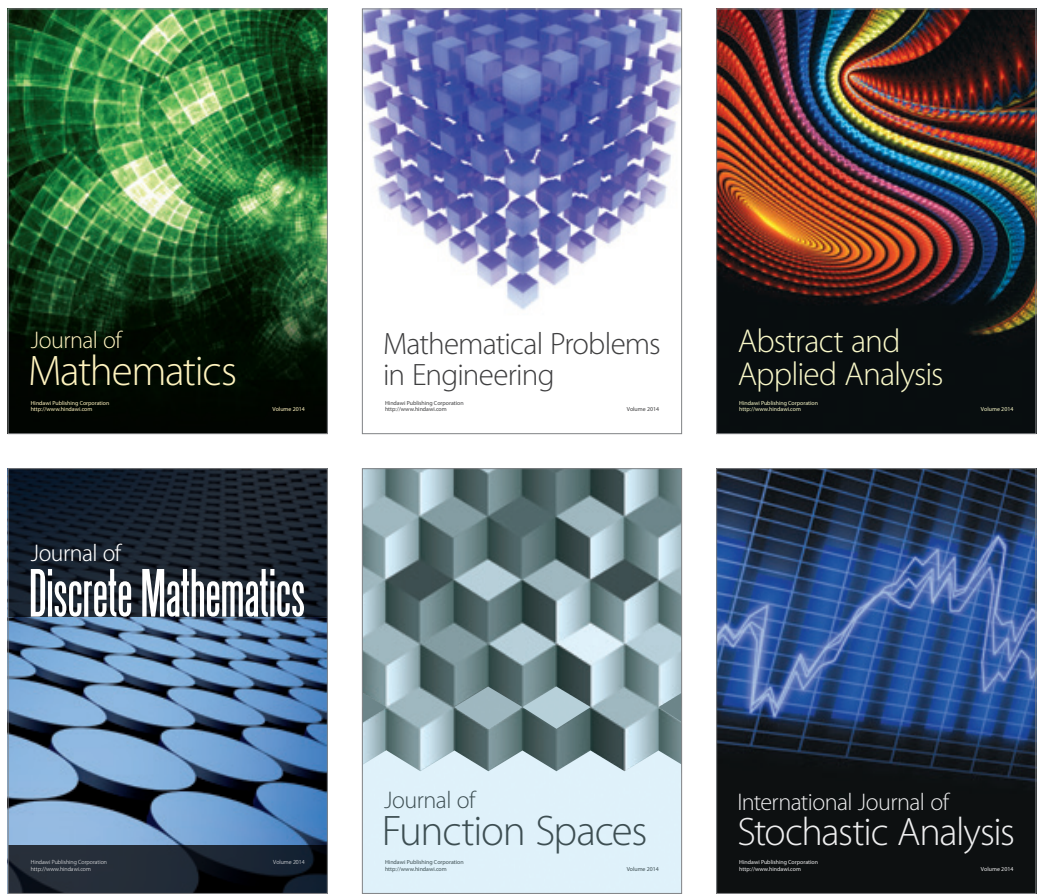

Journal of

Function Spaces

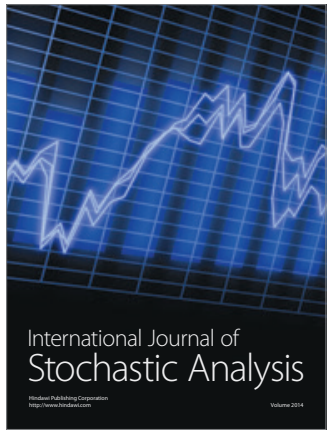

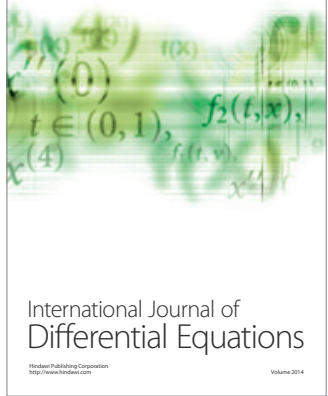
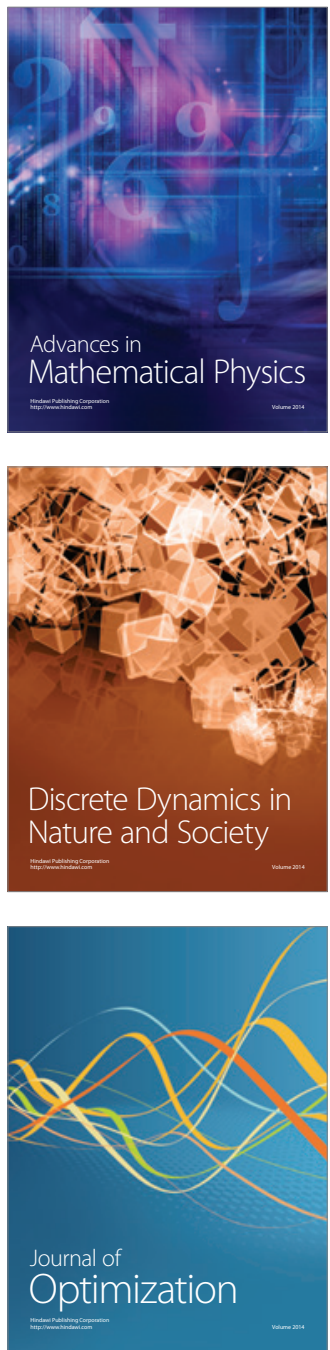